\title{
DE LA VISUALIZACIÓN A LA DEMOSTRACIÓN
}

\author{
Tomás Ortega del Rincón \\ Cristina Pecharromán Gómez \\ Universidad de Valladolid
}

RESUMEN: El propósito de la investigación es valorar el razonamiento, de un grupo de profesores de matemáticas de Chile y un grupo de alumnos del máster de profesor de secundaria, sobre la garantía de la exactitud o no de varias construcciones geométricas de un pentágono regular inscrito en una circunferencia. El análisis de datos permite observar que, en general, no se utilizan los procedimientos de construcción para razonar sobre la exactitud y rigor de la construcción. En cambio, se dan justificaciones basada en percepciones sensoriales, recuerdos o se utilizan procedimientos aritméticos que no llegan a coordinarse con la visualización del proceso de construcción y la propia construcción.

PALABRAS CLAVE: Visualización, demostración, pentágono regular, construcción exacta, didáctica de las matemáticas.

\section{FROM VISUALIZATION TO DEMOSTRATION}

ABSTRACT: The purpose of this research is to assess the reasoning of a group of Chilean mathematics teachers and a group of students of a "High School Teacher" master's degree, about the assurance of the accuracy, or not, of some geometric constructions of a regular pentagon inscribed in a circumference. Data analysis reveals that, in general, they do not use the construction procedures to argue about the accuracy and rigor of the construction. Instead of it, they give justifications based on sensory perceptions, memories or they use arithmetic procedures that fail to coordinate with the visualization of the construction process and geometric construction itself.

KEYWORDS: Display, proof, regular pentagon, exact construction, didactics of mathematics.

Recibido: 11/03/2015

Aceptado: 15/06/2015 
Correspondencia: Tomás Ortega del Rincón, Universidad de Valladolid, Facultad de Educación, Paseo de Belén 1, 47011 Valladolid. Email: ortega@am.uva.es.

\section{MarCos teóricos}

Interpretando a Schoenfeld (2010), consideramos que el conocimiento matemático de una persona es la información que tiene y que puede utilizar para resolver problemas y tareas matemáticas. Según este autor, este conocimiento no tiene por qué manifestarse como correcto, ya que, en múltiples casos, se producen razonamientos incorrectos que conducen a soluciones erróneas.

Hay varios modelos que caracterizan el conocimiento matemático de los profesores. El primero que consideramos fue creado por Shulman (1986) y consta de tres componentes: Subject Matter Knowledge (SMK), Pedagogical Content Knowledge (PCK) y Curricular Knowledge (CK). El conocimiento de contenido matemático (SMK), hace referencia a la cantidad y a la organización del conocimiento matemático en la mente del profesor (Shulman, 1986). El conocimiento pedagógico (PCK) hace referencia a toda la fenomenología didáctica que permite la enseñanza-aprendizaje del contenido matemático a través de la comunicación en el aula. Finalmente, el conocimiento curricular (CK) se refiere a la interpretación del contenido matemático del currículo.

En la investigación que nos ocupa, tiene especial relevancia el SMK, conocimiento que, desde la perspectiva profesional, ha despertado mucho interés en los últimos años, dando lugar a numerosos modelos de conceptualización. Así, Ball, Thames y Phelps (2008) propusieron el modelo Content Knowledge for Teaching (MKT) que distingue tres categorías dentro del conocimiento matemático que deben tener los profesores de matemáticas: Common Content Knowledge (CCK), que es el conocimiento matemático que ha alcanzado cualquier persona y, en relación a la profesión de profesor, el conocimiento que necesita saber el profesor para enseñar, justificar, relacionar y utilizar el contenido que enseña; Specialized Content Knowledge, que hace referencia a los conocimientos y habilidades didácticas que debe tener un profesor para transmitir el contenido matemático y promover su aprendizaje; Horizont Content Knowledge (HCK), que, aunque es difícil de precisar (Jakobsen, Thames y Ribeiro, 2013), se podría entender como las conexiones que se pueden establecer entre el contenido matemático y la interpretación de estas conexiones desde un puntos de vista curricular: interpretando el currículo de forma horizontal (en cada curso) y vertical (entre los distintos cursos) y entre distintas áreas de la matemática. Ball, Thames y Phelps (2008) consideran que el conocimiento de contenido curricular forma parte del conocimiento del contenido pedagógico, conocimiento necesario para el ejercicio de la profesión de profesor. Este conocimiento tiene menor influencia en nuestra investigación, por tanto, no lo analizamos.

Con el referente de este marco teórico, y la matemática asociada al pentágono regular (que aparte de que en $6^{\circ}$ de Educación Primaria se enseñan los polígonos regulares, el contenido elemental también está incluido en $2^{\circ}$ y $3^{\circ}$ Enseñanza Secundaria. También está asociado a la proporción áurea, a giros, a simetrías y a las raíces de números complejos), queremos establecer qué conocimientos matemáticos poseen los individuos de la muestra, qué conocimientos y habilidades profesionales tienen, y 
qué conexiones pueden establecer. En concreto, formulamos las siguientes preguntas de investigación: ¿Forma parte del conocimiento común del profesorado el contenido matemático asociado al pentágono regular? ¿Está especializado el profesorado en el conocimiento de este contenido? ¿Cómo es el horizonte de su conocimiento en relación a este contenido?

Por otra parte, Duval (1995) considera que el procedimiento de construcción geométrica de los objetos geométricos es muy útil para observar propiedades de estos objetos y establecer relaciones entre los elementos que los configuran. Asimismo, considera que los razonamientos analíticos relativos a estas interpretaciones (propiedades y relaciones) se deben producir asociados al procedimiento de construcción de la representación gráfica. Es decir, ¿se puede Ilegar a producir una coordinación entre la representación gráfica y aritmética o algebraica a través del procedimiento de construcción del objeto geométrico? Sin embargo, Duval (1995) indica que esta coordinación, está lejos de ser adquirida por los alumnos universitarios.

El desarrollo de esta coordinación está relacionado con la visualización, concepto ampliamente tratado por diversos autores, entre los que destacamos a Presmeg (1997, 2006) y a Fernández (2013). Sin embargo, en este trabajo seguimos a de Guzmán (1996: 3), quien afirma que visualización es "una interpretación de lo que se presenta a nuestra contemplación que solamente podremos realizar eficazmente si hemos aprendido a leer adecuadamente el tipo de comunicación que la sustenta". Es decir, tienen que saber interpretar el contenido matemático asociado a la percepción visual de la figura representada. Atendiendo a las ideas de Duval y de Guzmán, nos preguntamos si estos profesores y estos alumnos del máster de Educación Secundaria habrán adquirido esta coordinación y si se producirán visualizaciones en el sentido que señala de Guzmán.

Las preguntas que se han planteado, fundamentadas en el marco teórico, orientan el desarrollo de la investigación, y las reflexiones sobre las ideas de Duval y de Guzmán sobre la interpretación de los objetos geométricos y su procedimiento de construcción, permiten plantear los siguientes objetivos de investigación:

O1. Averiguar si el contenido matemático asociado al pentágono regular forma parte del conocimiento común del profesorado.

El Conocimiento Especializado del Contenido (SCK) es el conocimiento necesario para transmitir el contenido matemático en un contexto de enseñanza (por ejemplo, elección de representaciones y su secuenciación, mostrar los usos del contenido, ejemplificar procedimientos...). En relación a esta investigación consideramos que este conocimiento también abarca la coordinación entre las representaciones gráfica y aritmética. Por tanto, formulamos el segundo objetivo:

O2. Detectar si los individuos de la muestra han adquirido la coordinación entre la representación gráfica y numérica del procedimiento de construcción del pentágono regular.

El Conocimiento en el Horizonte Matemático, o relaciones en el contenido matemático curricular, orienta el tercer objetivo de la investigación:

O3. Indagar si los individuos de la muestra saben establecer conexiones entre el pentágono regular y otros conceptos matemáticos relacionados con él. 


\section{AnTECEDENTES}

Aunque no se han encontrado unos antecedentes cercanos o directamente relacionados con los objetivos que dirigen esta investigación, en este apartado recogemos algunos estudios relativos al pentágono regular.

Pritchard (2013) estudia la presencia de la sucesión de Fibonacci en el pentágono regular; Presmeg (2011) utiliza la geometría dinámica para analizar el pentágono regular; Eisenmann, Kopka, Ondrušová y Přibyl (2013) abordan la resolución de problemas; Miles y Pritchard (2009) analizan el contenido trigonométrico asociado al pentágono regular; finalmente, Bataille (2009) y Hofstetter (2013) se centran específicamente en métodos de construcción del pentágono regular.

Como se verá más adelante, en esta investigación se presentan tres procedimientos de construcción del pentágono regular, aparecen relaciones métricas y trigonométricas relativas este polígono, se plantean tareas que pueden considerarse problemas y, también, se utiliza un software dinámico para interpretar al pentágono regular y su procedimiento de construcción. Sin embargo, la orientación de esta investigación es muy distinta a la de los estudios reseñados.

\section{Metodología}

Se utiliza una muestra formada por dos grupos de individuos. El grupo 1 lo forman 29 profesores chilenos de Educación Secundaria que hicieron una Pasantía de Matemáticas (se codifican Pmn) en la Universidad de Salamanca durante el curso 2013-2014. El grupo 2 lo forman 12 alumnos del Máster de Profesor de Enseñanza Secundaria de la especialidad de Matemáticas (3 arquitectos, 4 ingenieros y 5 matemáticos), realizado en la Universidad de Valladolid durante el curso 2013-2014, (se codifican Ahk).

Se trata de una investigación cualitativa y, por tanto, no pretende que las conclusiones sean extrapoladas a las poblaciones de las muestras (Profesorado de Educación Secundaria de Chile y alumnos del Máster de Educación Secundaria).

Se realizan dos experimentaciones, es decir, se recogen datos de ambos grupos, pero en dos momentos diferentes. Para recoger los datos, se pasó en ambos grupos un test artesanal de siete ítems de respuesta abierta, relativos a las tres construcciones geométricas del pentágono regular que se presentaron. La corrección se realizó de forma artesanal interpretativa con el referente del marco teórico que aportan los contenidos matemáticos asociados al pentágono regular. Los datos se agrupan por afinidad y se presenta una discusión interpretativa de los mismos por bloques de afinidad.

En la docencia de ambos grupos, se construyeron con GeoGebra las tres construcciones geométricas que aparecen en el test, pero no se dio respuesta a las preguntas asociadas.

Todos los participantes cumplimentaron el test como tareas de los respectivos cursos de formación y las realizaron fuera del horario lectivo.

Para presentar los datos y su análisis, en primer lugar, se muestra el enunciado de la pregunta; en segundo lugar, el análisis de datos del grupo 1 y, en tercer lugar, el análisis de datos del grupo 2. 
El grupo 1 contestó al test trabajando principalmente por grupos, por lo que se disponen de 19 respuestas diferentes. Éstas fueron remitidas por correo electrónico. Las respuestas de estos alumnos se codifican por $\mathrm{PMN}$, donde $M=0,1$ y $N=0,1,2, \ldots$, 9. Los miembros del grupo 2 trabajaron de forma individual, por lo que se disponen de 12 respuestas, que se presentaron en papel. Éstas se codifican por ARS, con $R=0$, 1 y $S=0,1,2, \ldots, 9$.

\section{ANÁLISIS DE DATOS}

En lo que sigue, se presenta el análisis de los datos obtenidos a través del cuestionario, de los dos grupos que configuran la muestra, y se ejemplifica alguno de los datos mediante el escaneo de alguna respuesta.

\section{Pregunta 1}

Definición. Un polígono es regular si tiene sus lados y sus ángulos iguales.

Se ha construido el pentágono de la figura 1 así:

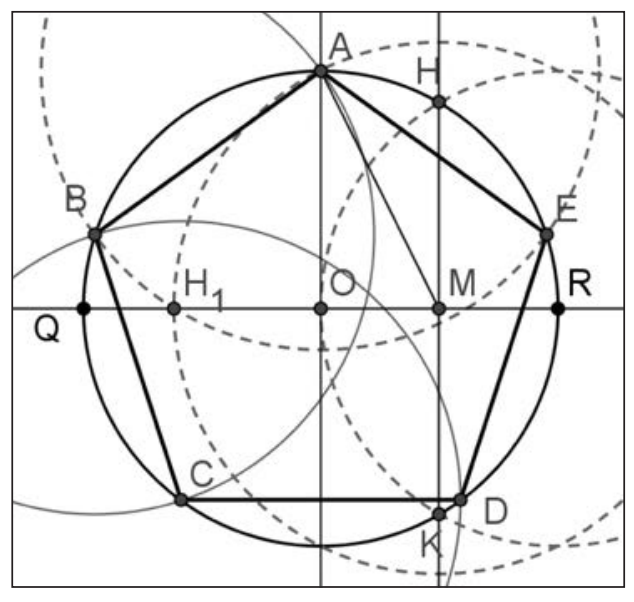

Figura 1. Construcción 1 del pentágono regular
a) Se dibuja la circunferencia de centro $\mathrm{O}$ y radio $\mathrm{OA}=\mathrm{OR}=1$.
b) Se dibuja el punto medio, $M$, de OR.
c) La circunferencia de centro $M$ y radio $M A$ corta al diámetro $Q R$ en $H_{1}$.
d) $\mathrm{AH}_{1}$ es la longitud del lado del pentágono inscrito.
e) Las intersecciones $\mathrm{B}$ y E de la circunferencia de partida con la circunferencia de centro $\mathrm{A}$ y radio $\mathrm{AH}_{1}$ son dos vértices del pentágono regular.
f) El pentágono se completa trazado sucesivos arcos de radio $A B$ con centros en $B$, para obtener $C$, en $C$ para obtener $D$, en D para obtener $E$ (que pre- sumiblemente coincidiría con el punto ya dibujado), y uniendo los vértices consecutivos. 


\section{Pregunta 1}

Explica si se ha establecido que los ángulos centrales son iguales.

El objetivo de este ítem es averiguar si los PPC y los AMM se dan cuenta de que en ningún paso de la construcción geométrica de la figura 1 se ha establecido la igualdad de los ángulos centrales.

Respuesta: No se tiene seguridad de que los ángulos centrales sean iguales, pues aunque la construcción ofrece lados iguales, la construcción no asegura que no pudiera haber un solapamiento o un hueco en el vértice $\mathrm{E}$ (el arco de centro $\mathrm{D}$ y radio $A B$, en principio, no tendría por qué cortar a la circunferencia dada en $E$ ).

Análisis de datos grupo 1: No se produce ninguna respuesta correcta.

Justificaciones de las respuestas de los alumnos de la pasantía

- El pasante P01 no escribe justificación alguna y el P26, basándose en una percepción visual, razona de forma incorrecta y realiza afirmaciones sin sentido que no justifican nada. Si entendemos que el ángulo del centro corresponde a $360^{\circ}$ dividido por 5 daría $72^{\circ}$, pero al observar la construcción tenemos que la relación entre la diagonal y el lado del pentágono regular es el número áureo. Por lo tanto no es un ángulo exacto (P26).

Respuestas que se basan en visualizaciones:

- Hacen una construcción errónea con GeoGebra. Con esta construcción, P05 observa distinta longitud de lados, y P20 y P27 obtienen ángulos interiores distintos.

- Realizan una construcción con GeoGebra y afirman que los ángulos centrales son iguales, pero no lo justifican.

- Una respuesta análoga a la anterior es la de P29, quien afirma que la construcción divide a la circunferencia en cinco arcos iguales.

- Finalmente, P19 y P25 construye con GeoGebra un pentágono impreciso, "que no se cierra".

Otras respuestas, a priori, consideran que el pentágono construido es regular y, por tanto, consideran que los ángulos centrales son iguales.

- Aquí se encuadran las respuestas de P09, P10, P17, P18, P22, P24. Estos profesores consideran que la figura 1 está formada por cinco triángulos isósceles iguales (afirman que son iguales por el desarrollo de la construcción) y concluyen que los ángulos centrales son iguales.

Cálculos numéricos:

- Considerando que r=1, P09 y P24 calculan la longitud del lado del pentágono,

$$
\overline{A N}=\sqrt{\frac{5-\sqrt{5}}{2}}
$$


- P16 y P21 calculan numéricamente del lado del pentágono y del ángulo central a través de la figura 1 (suponen que radio=1 y perciben que el pentágono está dividido en cinco triángulos isósceles iguales), indican que el número irracional obtenido se corresponde con $72^{\circ}$, pero no dicen por qué.

Análisis de datos grupo 2

- No hay respuestas totalmente correctas y, aunque algunos afirman que los ángulos son iguales, los razonamientos que aportan son erróneos.

Justificaciones incorrectas de las respuestas de los alumnos:

- La percepción sensorial de la construcción lleva al alumno A07 indicar que los ángulos centrales parecen iguales, pero no justifica por qué.

- Varios alumnos perciben que la propia construcción proporciona ángulos iguales: A01, A03, A10, A11. Otros dos alumnos, A09 y A12, también responden de forma afirmativa "porque la construcción ofrece un pentágono regular" y consideran que es una consecuencia de la regularidad. El alumno A02 da un paso más y escribe que no hay seguridad de que los ángulos sean iguales, porque no se ha comprobado la longitud del último lado, que se ha formado uniendo el último punto obtenido con el primero.

- Para el alumno A04 no son iguales pero el razonamiento es totalmente erróneo y alude al centro de la circunferencia.

No, una vez hallada la lougitud del lado del peutágono. 2e ha ido trastadaudo per la circunferancia sin establecer relación con el cectro de la misma, que en este ceso será el seutro del pentagono.

Figura 2. Respuesta alumno A04.

- Finalmente, los alumnos A05, A06, A08 y A12 afirman que los ángulos no son iguales porque en la construcción no se ha utilizado que los ángulos centrales sean iguales.

\section{Pregunta 2}

Explica si puedes tener la absoluta seguridad de que el pentágono es regular.

El objetivo de este ítem es averiguar las razones que dan los PPC y los AMM sobre la seguridad de sus respuestas a la pregunta anterior,

Respuesta: La construcción realizada no asegura que haya un cierre exacto, luego, en principio, con la única argumentación de la construcción realizada no se puede afirmar que los ángulos centrales sean iguales y, en consecuencia, el pentágono construido sea regular. 
Análisis de datos del grupo 1

- Sólo el profesor P29 responde correctamente y afirma que "la construcción no asegura que los ángulos centrales sean de $72^{\circ}$, por lo que se cerraría el polígono, entonces no se puede asegurar que sea regular".

Justificaciones incorrectas de las respuestas de los alumnos

- El pasante, P26, igual que en el ítem anterior, sigue un razonamiento incorrecto, y realiza afirmaciones carentes de sentido que no justifican nada.

- P01 muestra ahora su inseguridad, porque no tiene la certeza de que los ángulos interiores sean iguales.

- Varios de ellos se basan en la construcción realizada con GeoGebra. Así, P05, P20 y P27 afirman no estar seguros, porque en la construcción con GeoGebra no se observan lados (o ángulos) iguales. Por el contrario, P13, P23 y P28 se basan en una comprobación, ellos están seguros "porque en la construcción con GeoGebra se miden lados y ángulos iguales". P19, P25 son consecuentes con la construcción que han realizado antes con GeoGebra y, como es inexacta (la hacen mal), afirman que no proporciona un pentágono. También P09, P10, P16, P17, P18, P21, P22 y P24 afirman que están seguros, porque para ellos la construcción proporciona lados iguales y, entonces, los ángulos iguales.

El profesor P20 responde: "No, porque al realizar paso a paso el pentágono regular inserto en la circunferencia y medir los ángulos interiores no todos son iguales, es decir no miden $108^{\circ}$, por lo tanto los ángulos del centro no son iguales".

Análisis de datos grupo 2

- Todos los alumnos se basan en una u otra interpretación de la construcción. El alumno A01, en primer lugar, hace un razonamiento condicional, pero termina afirmando que "es regular si la línea poligonal se cierra en $E$, pues la construcción ofrece lados y ángulos iguales. A02, A05, A06 y A09 responden negativamente "porque el último lado se ha trazado uniendo puntos y no marcando el lado". Para otros cuatro alumnos, A03, A04, A08, A11 y A12, la seguridad procede de "la propia construcción, lados y ángulos iguales". Algo parecido escribe A07 "sí porque la construcción proporciona lados iguales. Finalmente, A10, cuestiona la exactitud de la construcción a la precisión de la herramienta, pero afirma que el proceso seguido es correcto "sólo se puede asegurar que el proceso seguido es correcto, no la ejecución, que depende de la precisión de herramientas".

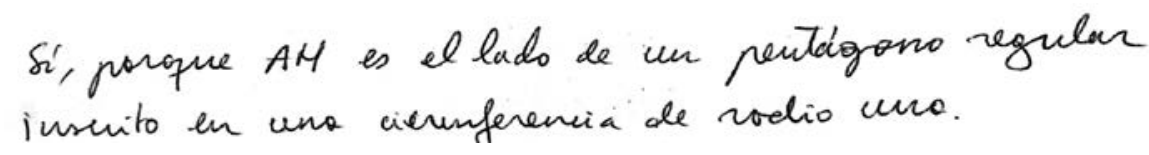

Figura 3. Respuesta alumno A11 


\section{Pregunta 3}

Explica si puedes tener la absoluta seguridad de que los lados se cierran exactamente en $\mathrm{E}$.

El objetivo de este ítem es averiguar las explicaciones que dan PPC y los AMM sobre el cierre de la poligonal construida. Es decir, si el arco de centro D y radio AB corta forzosamente a la circunferencia dada en $\mathrm{E}$ y ver si el número de respuestas afirmativas aumenta o disminuye respecto de la pregunta 2.

Respuesta: La construcción no asegura que el arco de centro D y radio AB corte forzosamente a la circunferencia dada en E.

\section{Análisis de datos grupo 1}

- El pasante P29 es el único que responde correctamente y escribe que "No se tiene la seguridad de que los lados se vayan a cerrar porque no se tiene la seguridad de que los ángulos valgan $72^{\circ}$.

\section{Justificaciones incorrectas de las respuestas de los alumnos}

- P01 sigue la tónica anterior y no escribe justificación alguna. Análogamente, P26 escribe un razonamiento incorrecto y carente de sentido justificativo.

- Un grupo numeroso de alumnos basan su respuesta en la "visualización". P05, P10, P13, P16, P17, P18, P20, P22, P23, P27 y P28. Repiten la construcción con GeoGebra y afirman que se cierran los lados. En este grupo también se pueden considerar las respuestas de P19 y P25, aunque estos profesores construyen con GeoGebra un pentágono que no se cierra y, en consecuencia, para ellos la respuesta negativa es obvia. Respuesta de P16: Se tiene absoluta seguridad de que los lados del pentágono se cierran por construcción hecha en GeoGebra paso a paso de la figura 1. Además por lo señalado en el punto 1 y 2 (anteriormente). La medida de cada uno de los lados del pentágono mide 1,18 aproximadamente cuando el $r=1$.

- Dos alumnos, P09 y P24 interpretan la figura 1 configurada por cinco triángulos isósceles iguales (por el desarrollo de la construcción). Concluyen que los ángulos centrales son iguales, todos de $72^{\circ}$, lo que justifica su cierre. Otros dos alumnos P16 y P21 realizan el cálculo numérico del lado del pentágono y del ángulo central apoyados en la construcción de la figura 1 (suponen radio=1 y pentágono dividido en cinco triángulos isósceles iguales). Indican que el número irracional obtenido se corresponde con $72^{\circ}$, pero no dicen por qué. Este valor del ángulo central les lleva a decir que el polígono se cierra.

\section{Análisis de datos grupo 2}

Aparte del alumno A12, que no hace justificación alguna, y del alumno A01 que respondió con razonamientos totalmente incoherentes, se presentan las siguientes respuestas, todas ellas con justificaciones erróneas:

- Sólo hay un alumno, el A09, que indica que la construcción no se deduce que los lados se cierren, pero la justificación que escribe es incorrecta "porque el 
método gráfico no tiene precisión infinita". También, el alumno A06 asocia la exactitud a la precisión de las herramientas de dibujo a la precisión que ofrece GeoGebra como si las construcciones hechas con ordenador fueran siempre correctas y no así las que se hacen con regla y compás:

\section{Si, porques se ha becko con un programa informático y} halla de forma exacta el pto de üiterseccioú de los lades DE y EA. Si lo hubiéramos hecho con regla y compás uO teudrianos absoluta seguridad por los pequễos errores que se pueder cometer ue la construcción.

Figura 4. Respuesta alumno A06

- Otros alumnos consideran como punto de partida que el pentágono es regular: A07 afirma que "el pentágono es regular, luego los lados se cierran" y A03 considera de entrada que "los ángulos centrales son iguales y, por tanto, el pentágono se cierra".

- Finalmente, A02, A04, A05, A08, A10 y A11 aseguran que la poligonal se cierra "porque así se ha impuesto en la construcción".

\section{Pregunta 4}

La figura 2 se ha hecho con GeoGebra considerando que $\cos \left(72^{\circ}\right)=\frac{\sqrt{5}-1}{4}$.

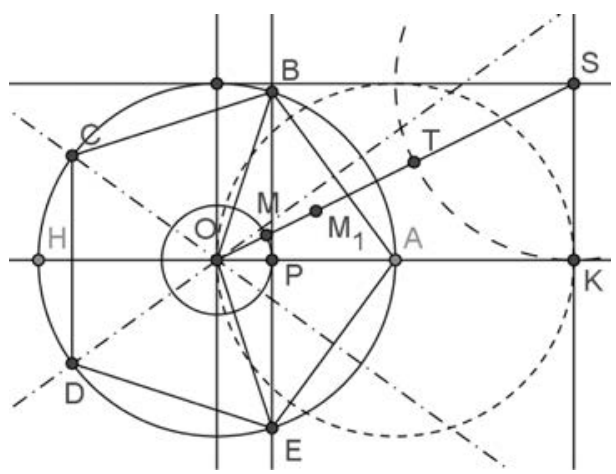

Figura 5. Construcción 2 del pentágono regular

a) Se dibuja la circunferencia de radio $\mathrm{OA}=1$

b) Se construye el triángulo rectángulo de catetos $\mathrm{OK}=2$ y $\mathrm{KS}=1$. La longitud del segmento OT es $O T=\sqrt{5}-1$ 
C) Se dibuja el punto medio, $M_{1}$, del segmento OT y $O M_{1}=\frac{\sqrt{5}-1}{2}$

d) Se dibuja el punto medio, $M$, de $\mathrm{OM}_{1}$, y $O M=\frac{\sqrt{5}-1}{4}$.

e) Con la circunferencia de centro $\mathrm{O}$ y radio $\mathrm{OM}$ se construye

$$
O P=\frac{\sqrt{5}-1}{4}=\cos \left(72^{\circ}\right)
$$

f) Considerando que $\mathrm{A}$ es un vértice, se traza la recta perpendicular a $\mathrm{AO}$ por $\mathrm{P}$ y la intersección de ésta con la circunferencia de partida determina los vértices $B$ y E del pentágono $\left(\mathrm{AOB}=\mathrm{EOA}=72^{\circ}\right)$.

g) Los otros dos vértices se determinan por simetría.

Explica si ahora es seguro que ABCDE es un pentágono regular.

Es objetivo de esta pregunta es analizar si tanto los PPC como los AMM son conscientes de que, aceptando como verdadera la hipótesis de partida, esta construcción geométrica sí que es exacta.

Respuesta: Sí, ahora es seguro que el pentágono así construido es regular, porque con este procedimiento se construyen cinco triángulos isósceles iguales. Ambos tienen iguales dos lados (son radios) y el ángulo comprendido.

\section{Análisis de datos grupo 1}

Solo hay una respuesta que se puede considerar correcta la del pasante P29. Éste afirma que se tiene la certeza de que el pentágono es regular porque se parte de la construcción del ángulo de $72^{\circ}$, para a partir de él obtener el lado del pentágono (No argumenta por qué los lados son iguales).

Justificaciones erróneas de las respuestas de los alumnos

- Los profesores P01, P09 y P24 afirman que sí, "porque con cinco ángulos de $72^{\circ}$ se cubre toda la circunferencia". Sin embargo, no utilizan el dato del enunciado (el valor de $\cos \left(72^{\circ}\right)$ ), no parece que reconozcan que ahora se conoce primero el ángulo y luego se determina el lado partiendo de su proyección. Se presenta la respuesta de P09:

Por demostración del punto 1. Podemos asegurar que: $\overline{A B}=\overline{B C}=\overline{C D}=\overline{D E}=\overline{E A}$. $\mathrm{Y}$, al tener $\angle A O B \cong \angle A O E \cong \angle B O C \cong \angle C O D \cong \angle D O E=72^{\circ}$, y que los triángulos $\triangle A O B \cong \triangle A O E \cong \triangle B O C \cong \triangle \cong C O D \cong \triangle D O E$ son isósceles, podemos asegurar que los $\angle A B C \cong \angle B C A \cong \angle C D E \cong \angle D E A \cong \angle E A B$ y miden $108^{\circ}$ por lo tanto el pentágono $A B C D E$ es regular.

- Aparecen otras respuestas muy dispares: P05 y P26 aseguran que el hecho de que $\cos \left(72^{\circ}\right)$ sea un número irracional no asegura que el pentágono sea regular; P10, P16, P17, P21 y P22 no contestan a la pregunta y repiten la construcción que aparece el enunciado; tampoco responden a la pregunta formulada P19, P20, P25 y P27, pero, basándose en la percepción visual, suponen que el pen- 
tágono es regular y calculan la razón entre la diagonal y el lado mediante semejanza de triángulos; por otra parte; P18, conoce que la razón entre la diagonal y el lado es el número de oro y hace un cálculo numérico distinto al que ofrece el enunciado, a partir de un triángulo áureo, hace un razonamiento inverso al constructivo, pero no le permite observar la relación entre el ángulo de $72^{\circ}$ y el lado del pentágono y, por tanto, no, muestra un razonamiento en el que determina sen $\left(18^{\circ}\right)$, pero no justifica su respuesta, que se presenta a continuación:

$\mathrm{Si}$ es seguro porque en el pentágono regular, trazamos desde un vértice $\mathrm{B}$ las diagonales $\mathrm{BE}$ y $\mathrm{BD}$ dónde nos queda un triángulo áureo menor determinado por estas y el lado DE. Se traza una altura desde el punto B al punto medio DE formando dos triángulos rectángulos con lo siguiente:

La hipotenusa es la diagonal del pentágono, el ángulo menor es de $18^{\circ}$ y su cateto opuesto es la mitad del lado del pentágono, el ángulo agudo mayor mide $72^{\circ}$.

Aplicando la definición del seno y coseno obtenemos que: $\frac{B E}{D E}=\frac{1+\sqrt{5}}{2}$

Sea $M$ punto medio de DE: $\operatorname{sen}\left(18^{\circ}\right)=\cos \left(72^{\circ}\right)=\frac{E M}{B E}=\frac{D E / 2}{B E}=\frac{\sqrt{5}-1}{4}$

- P13 y P28 escriben un razonamiento sin sentido y, finalmente, P23 afirma sin justificación alguna que la construcción permite obtener lados iguales.

Análisis de datos grupo 2

Aunque no terminan el razonamiento $\left(5^{*} 72^{\circ}=360^{\circ}\right)$, se puede considerar que los pasantes A01, A02, A03, A05, A08, A11 y A12 responden correctamente y lo justifican mediante la igualdad de arcos: "Sí, porque se puede trasladar un arco de $72^{\circ}$ cinco veces exactamente".

Justificaciones incorrectas de las respuestas de los alumnos

- A09 realiza un razonamiento totalmente incoherente basado en una comprobación mediante cálculos aproximados:

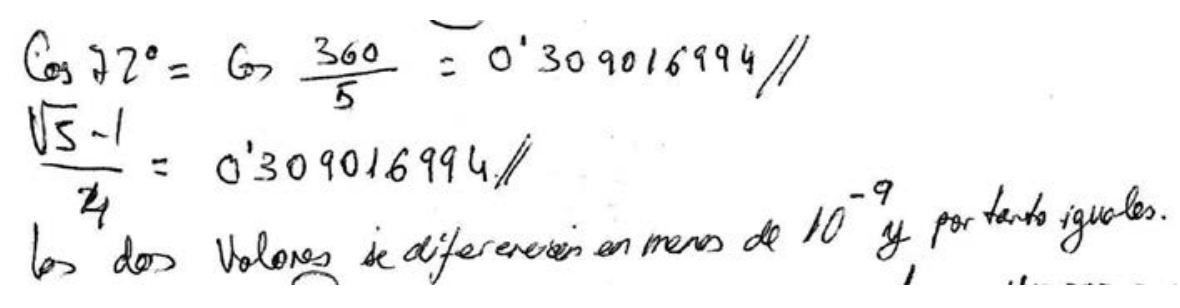

Figura 6. Respuesta alumno A09

- A06, A04, A08 y A10 afirman que sí, que el pentágono es regular, porque se obtienen lados iguales (pero no justifican por qué). Finalmente, A07 calcula el valor de los ángulos interiores del pentágono aplicando el teorema del seno con el ángulo de $72^{\circ}$, pero sólo obtiene aproximaciones. 


\section{Pregunta 5}

Calcula cuánto mide el lado del pentágono regular construido en las figuras anteriores, sabiendo que el radio de la circunferencia circunscrita mide $1 \mathrm{~m}$.

El objetivo de este ítem es averiguar la capacidad de cálculo de los PPC y los AMM. Concretamente, comprobar si llegan a determinar la longitud del lado del pentágono regular inscrito en una circunferencia de radio la unidad a partir de la visualización de las figuras presentadas.

Respuesta: $\overline{A B}=\sqrt{\frac{5-\sqrt{5}}{2}}, \overline{A B}=2 \operatorname{sen}\left(36^{\circ}\right), \overline{A B}==1,17557 \mathrm{~m}$ es una aproximación numérica.

Análisis de datos grupo 1

- Se producen 11 respuestas correctas y bien justificadas, pero la argumentación no siempre es la misma: P16, P21 y P26 obtienen el radical correcto y lo justifican aplicando el teorema de Pitágoras a los triángulos rectángulos de la figura 1; Asimismo, P09, P2 4 y P29 obtienen el valor radical correcto, pero en este caso utilizan el teorema del coseno en uno de los cinco triángulos isósceles en que queda dividido el pentágono, sustituyendo $\cos \left(72^{\circ}\right)$ por su valor radical; finalmente, P18, P19, P20, P25 y P27 hallan correctamente el valor trigonométrico aproximado correcto y lo justifican aplicando trigonometría básica en uno de los cinco triángulos isósceles en que queda dividido el pentágono. Se presenta la respuesta de P19 que acompaña el gráfico de la derecha:
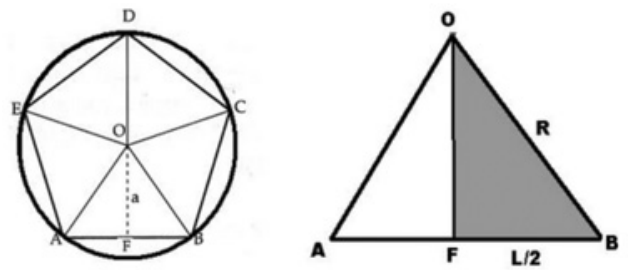

Figura 7. Respuesta alumno P19

$\mathrm{ABCDE}$ pentágono regular, $\mathrm{AB}=\mathrm{BC}=\mathrm{CD}=\mathrm{DE}=\mathrm{EA}=\mathrm{L}(\mathrm{Lado}), \mathrm{OA}=\mathrm{OB}=\mathrm{OC}=\mathrm{OD}=\mathrm{OE}=\mathrm{R}$ (Radio) $, \angle \mathrm{AOB}=\angle \mathrm{BOC}=\angle \mathrm{COD}=\angle \mathrm{DOE}=\angle \mathrm{EOA}=72^{\circ},<\mathrm{FOBO}=36^{\circ}, \operatorname{sen}\left(36^{\circ}\right)=\frac{L / 2}{R}$ $\operatorname{Rsen}\left(36^{\circ}\right)=\mathrm{L} / 2 \Rightarrow \mathrm{L}=2 \operatorname{Rsen}\left(36^{\circ}\right)$, si $\mathrm{R}=1$ entonces $2 \operatorname{sen}\left(36^{\circ}\right)=\mathrm{L}$

Justificaciones incorrectas o respuestas erróneas

- Tanto P01, que utiliza el valor de $\cos \left(72^{\circ}\right)$ como P05 y P23, que parece que utilizan GeoGebra, obtienen resultados numéricos incorrectos y sin justificar; 
P13 y P28 obtienen un resultado incorrecto mediante un proceso de cálculo con radicales incorrecto; finalmente P10, P17 y P22 escriben el radical correcto, pero no aportan ninguna justificación.

\section{Análisis de los datos del grupo 2}

Todas las respuestas son correctas y están bien justificadas. Sin embargo, aportan varios procesos de justificación diferentes y obtienen expresiones numéricas diferentes:

- A01, A03, A05, A06, A11 y A12 obtienen un valor trigonométrico correcto y aplican de forma justificada trigonometría básica en uno de los cinco triángulos isósceles iguales en que queda dividido el pentágono.

- A01, A02, A03 y A04 hallan el radical correcto, aplicando el teorema del coseno en uno de los cinco triángulos isósceles iguales en que se puede dividir el pentágono. Sustituyen el valor de $\cos \left(72^{\circ}\right)$ por su expresión radical.

- A02 calcula el valor decimal correcto lo justifica aplicando el teorema de Pitágoras en figura 1, considerando valores decimales.

- A04 y A11 obtienen el valor radical correcto y lo justifican aplicando el teorema de Pitágoras en figura 1 y considerando valores radicales.

- A08 obtiene el valor radical correcto y lo justifica aplicando el teorema de Pitágoras en figura 2 y tomando valores radicales; A09 sigue el mismo proceso, pero utiliza valores decimales y obtienen una aproximación decimal:

- A07 utiliza datos trigonométricos y el teorema de Pitágoras en el triángulo rectángulo APB de la figura 2 y obtiene el valor trigonométrico correcto; A07 utiliza el mismo proceso, pero usa cálculos con decimales y obtiene un valor decimal aproximado. Se presenta el escaneada la respuesta de A07:

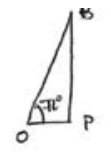

$$
\begin{aligned}
O B & =1 \text { u. } \quad O A=O P+P A \\
O P & =\cos 72^{\circ} \quad P A=O A-O P \\
P B & =\operatorname{sen} 72^{\circ} \quad P A=1-\cos 72 \\
\text { Por el th de Pitagoras, } & \\
(A B)^{2} & =(P A)^{2}+(P B)^{2}=\left(1-\cos 72^{\circ}\right)^{2}+\operatorname{sen}^{2} 72^{\circ}= \\
& =1+\cos ^{2} 72^{\circ}-2 \cos ^{\circ}+\operatorname{sen}^{2} 72^{\circ} \\
& =1+\cos ^{2} 72^{\circ}+\operatorname{sen}^{2} 72^{\circ}-2 \cos 72^{\circ}=2-2 \cos 72^{\circ} \\
& =2\left(1-\cos 72^{\circ}\right) \\
A B=\sqrt{2\left(1-\cos 72^{\circ}\right)} & u .
\end{aligned}
$$

Figura 8. Respuesta alumno A07

- A10 calcula un valor trigonométrico correcto y lo justifica aplicando el teorema del coseno en triángulo $\mathrm{AH}_{1} \mathrm{M}$ de la figura 1 y en la figura 2.

\section{Pregunta 6}

Se presenta la figura 3, y se describe cómo se ha construido el pentágono regular partiendo de la longitud del lado $A B=1$. 


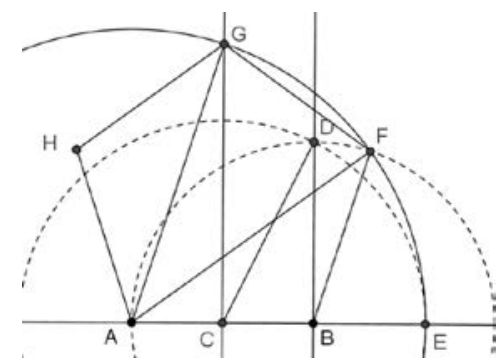

Figura 9. Construcción 3 del pentágono regular

a) Se dibuja el punto medio, C, de AB y la perpendicular a AB por B.

b) $\mathrm{BD}=1 \mathrm{y}$, por tanto, el segmento $\mathrm{CD}$ mide $\frac{\sqrt{5}}{2}$.

c) El segmenta AE mide $\frac{1+\sqrt{5}}{2}$.

d) La intersección de la circunferencia de centro $B$ y radio $A B$, y la circunferencia de centro $\mathrm{A}$ y radio $\mathrm{AE}$ es el tercer vértice.

e) EL cuarto vértice, G, es la intersección de la mediatriz de AB y la circunferencia de centro $A$ y radio $A E$.

f) $\mathrm{H}$ es la intersección de las circunferencias de centros $\mathrm{A}$ y $\mathrm{G}$, y radios $\mathrm{AB}$.

Se consideran las figuras 1,2 y 3, y se pide que calculen cuánto mide el radio de la circunferencia circunscrita si el lado del pentágono regular, $\mathrm{ABFGH}$, es la unidad.

El objetivo de este ítem es comprobar si se producen modificaciones respecto de las respuestas anteriores en este proceso de cálculo que es inverso del anterior y qué novedad aporta la figura 3.

Respuesta: $r=\sqrt{\frac{2}{5-\sqrt{5}}}, r=\frac{1}{2 \operatorname{sen}\left(36^{\circ}\right)}$, valor decimal aproximado: 0,85

Análisis de los datos del grupo 1

- Se producen 12 respuestas correctas justificadas: P05, P18, P19, P20, P26 y P27 obtienen el valor correcto en forma trigonométrica y lo justifican mediante trigonometría básica en uno de los cinco triángulos isósceles en que queda dividido el pentágono regular; los pasantes P09, P16, P21, P2 4 y P25 escriben el valor correcto en forma radical y lo justifican calculando el lado en función del radio aplicando el teorema de Pitágoras en la figura 1; finalmente, P29 escribe correctamente el valor y lo expresa también en forma radical, pero lo justifica aplicando el teorema del coseno en uno de los cinco triángulos isósceles que componen el pentágono regular.

\section{Justificaciones incorrectas o respuestas erróneas}

- El pasante P01 no contesta y el P23 da una respuesta carente de sentido. Finalmente, hay cinco respuestas, las dadas por P10, P13, P17, P22 y P28, que expresan en forma radical el valor correcto, pero no lo justifican. Así, P28 escribe lo siguiente: 
Dado que: $\frac{10 r^{2}-2 \sqrt{5} r}{4}=l, 10 r^{2}-2 \sqrt{5} r^{2}=4,5 r^{2}-\sqrt{5} r^{2}=2, r^{2}=\frac{2}{5-\sqrt{5}}, r=\sqrt{\frac{2}{5-\sqrt{5}}}$

Análisis de los datos del grupo 2

- Se producen 10 respuestas correctas justificadas, pero las expresiones numéricas o las justificaciones no son uniformes y se produce una casuística muy variada: Así, A10 escribe el valor correcto en forma radical correcto y lo justifica aplicando el teorema de Pitágoras a la figura 1; el pasante A03 utiliza la figura 3 y los datos asociados a ella, y expresa el valor correcto en forma radical en función de la tangente de $36^{\circ}$. A05 y A08 utilizan también la figura 3 y, con los datos asociados a ella, escriben el valor correcto en función del número de oro; también utilizan la figura 3 A04 y A06, que obtienen el valor correcto en forma trigonométrica mediante trigonometría básica; asimismo, A10 y A07 aplican trigonometría básica en uno de los triángulos isósceles iguales en que queda dividido el pentágono (figura 1) y hallan correctamente el valor en forma trigonométrica, pero el primero complica el proceso con teorema de Pitágoras; finalmente, A02 y A11 escriben el valor correcto en forma decimal y aplican el teorema del seno en uno de los triángulos isósceles iguales en que queda dividido el pentágono de la figura 2.

Justificaciones de las respuestas de los alumnos

- Los alumnos A12 y A09 obtienen sendos valores erróneos: el primero, que considera el resultado del ítem siguiente, sigue un proceso numérico correcto, pero construye dos figuras no relacionadas y supone que la longitud del lado es la unidad; el segundo porque, aunque elige un proceso adecuado basado en aplicar trigonometría básica en uno de los triángulos isósceles iguales en que queda dividido el pentágono regular, considera un valor angular equivocado. Se presenta escaneada la solución de A12:
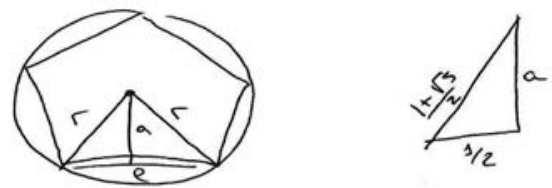

$$
\begin{aligned}
& \left(\frac{1+\sqrt{5}}{2}\right)^{2}=a^{2}+\left(\frac{1}{2}\right)^{2} \\
& \frac{(1+\sqrt{5})^{2}}{4}=a^{2}+\frac{1}{4} \\
& \frac{1+5+2 \sqrt{5}}{4}=\frac{4 a^{2}}{4}+\frac{1}{4} \\
& 1+5+2 \sqrt{5}-1=4 a^{2} \\
& 5+2 \sqrt{5}=a^{2} \rightarrow a=\sqrt{\frac{5+2 \sqrt{5}}{4}}
\end{aligned}
$$

Figura 10. Respuesta alumno A12 


\section{Pregunta 7}

Calcula la razón entre la diagonal y el lado del pentágono regular.

El objetivo de este ítem es averiguar hasta qué punto los alumnos Ilegan a establecer la razón aurea de forma justificada utilizando visualizaciones y construyendo las correspondientes relaciones numéricas.

Respuesta: $\Phi$

Análisis de los datos del grupo 1

- Se producen 6 respuestas correctas y justificadas: la primera es del pasante P18, que obtienen el valor correcto en forma trigonométrica y aplica trigonometría básica en el triángulo isósceles $A B F$ (figura 3), con $A B=1 \mathrm{~cm} ; \mathrm{P} 19$, P20, P25, P27 y P29 hacen una deducción teórica a partir de la semejanza de triángulos del pentágono regular.

\section{Justificaciones incorrectas o respuestas erróneas}

- Uno de los pasantes, P01, no contesta; también aparecen varias respuestas sin justificar (P05, P13, P23 y P28); hay razonamientos incompletos en procesos numéricos, P26, o en trigonométricos, P09 y P24; finalmente, cinco pasantes calculan la razón aplicando radicales, pero no justifican el valor radical de la diagonal (P10, P16, P17, P21 y P22). Se presenta la solución aportada por P21, quien, además, utiliza una relación conocida del número áureo para obtener dicho número. Como el lado del pentágono mide $\sqrt{\frac{5}{2}-\frac{\sqrt{5}}{2}}$ y la diagonal $\frac{\sqrt{10+2 \sqrt{5}}}{2}$ (esta última medida resulta del triángulo rectángulo OBP). Es así, como se puede plantear la razón entre ambas medidas, resultando lo siguiente:

$$
\begin{aligned}
& \frac{\frac{\sqrt{10+2 \sqrt{5}}}{2}}{\frac{\sqrt{5-\sqrt{5}}}{\sqrt{2}}}=\frac{\sqrt{10+2 \sqrt{5}}}{\sqrt{2} \cdot \sqrt{2}} \cdot \frac{\sqrt{2}}{\sqrt{5-\sqrt{5}}}=\frac{\sqrt{2}(\sqrt{5+\sqrt{5}}}{\sqrt{2}(\sqrt{5-\sqrt{5}}}=\sqrt{\frac{(5+\sqrt{5})(5+\sqrt{5)}}{25-5}}=\sqrt{\frac{3+\sqrt{5}}{2}} \\
& \sqrt{\frac{2}{2}+\frac{1+\sqrt{5}}{2}}=\sqrt{1+\phi}=\sqrt{\phi^{2}}=\phi \quad \text { Recordando que } 1+\varphi=\varphi^{2}
\end{aligned}
$$

\section{Análisis de los datos del grupo 2}

- Excepto el alumno A05 que utiliza como hipótesis la conclusión a la que tiene llegar (Conoce a priori el resultado y realiza una división de segmento asociada a número áureo, el resto emite respuestas correctas: unos calculando directamente las longitudes del lado y de la diagonal a partir de la figura 3 (A01, A02, A04, A08 y A11) y, otros, deduciendo la razón a partir de la semejanza de triángulos en pentágono regular (A03, A06, A07, A09, A10 y A12). Concretamente A10 considera "triángulos homotéticos" como sigue: 
Constuminos la siguiente relacion de triangulos homotéticos.
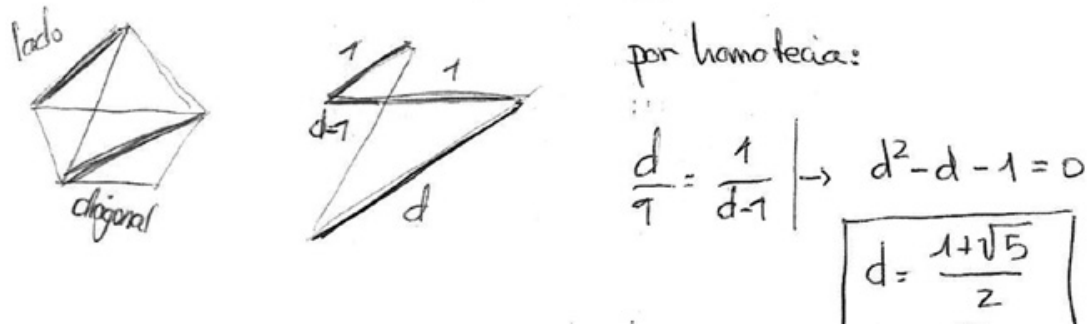

Figura 11. Respuesta alumno $A 10$

\section{Conclusiones}

El análisis de datos pone de manifiesto respuestas muy variadas pero, en general, se observa que no tienen lugar razonamientos o interpretaciones que manifiesten la formalización del conocimiento matemático relativo al pentágono regular y su construcción, en este caso, a través de la coordinación de las representaciones gráfica y analítica.

Los alumnos tienen ciertos conocimientos matemáticos (métricos, trigonométricos, gráficos) sobre el pentágono regular y el procedimiento exacto de construcción pero, en general, no aciertan a justificar la exactitud del procedimiento de construcción del pentágono regular mediante procedimientos aritméticos o algebraicos que se coordinen con la representación visual. Además, sustituyen razonamientos por evocaciones. Por tanto, no se alcanza la coordinación gráfico-aritmética asociada al pentágono regular y sus propiedades, algo que ya observó Duval, como se señala en el marco teórico.

Por otra parte, algunos individuos de la muestra sí que establecen conexiones con otros contenidos matemáticos presentes en el objeto matemático que tratamos, como relaciones métricas, trigonométricas, el número áureo, homotecias...

En ambos grupos, se representan los resultados numéricos en forma trigonométrica y radical, pero en el segundo no sustituyen razonamientos deductivos por mediciones, luego alcanzan un conocimiento matemático más desarrollado. Sin embargo, los errores que cometen unos y otros, y la gran variedad de justificaciones incorrectas observadas, ponen de manifiesto que no hay una coordinación entre las representaciones gráficas y numéricas, y que la visualización tal y como se ha concebido en el marco teórico está lejos de ser alcanzada.

Otra información de interés que se deriva del análisis de datos y corrobora las conclusiones expuestas es la siguiente:

- Algunos profesores del grupo de pasantía elaboran (repiten las dadas) construcciones con GeoGebra que fundamentan sus razonamientos a través de percepciones sensoriales, otros presentan procesos matemáticos de cálculos directos basados en las relaciones aritméticas de los segmentos que componen las figuras a través de visualizaciones, otros utilizan conocimientos de trigonometría (teoremas del seno y del coseno), otros se basan en semejanzas y otros, incluso, parten de lo que deben establecer, lo que prueba que el con- 
tenido asociado al pentágono regular forma parte del conocimiento común del profesorado y del horizonte matemático. Sin embargo, no se puede afirmar que este conocimiento forme parte del conocimiento especializado, ya que no logran establecer justificaciones adecuadas, incluso cometen errores de cálculo. Incluso, algunos pasantes hacen construcciones inexactas con GeoGebra que utilizan para sustituir los razonamientos por comprobaciones basadas en medidas de longitudes. Llama la atención que ningún alumno de este grupo utilice la figura 3 del cuestionario para hacer sus razonamientos. Parece que prefieren utilizar la figura presumiblemente más conocida (la figura 1, que es el procedimiento más habitual) o aquella que tiene una relación más directa con procesos más repetidos en el aula (la figura 2 tiene una relación directa con la trigonometría). La visualización de la primera figura influye más en la elección de un procedimiento aritmético, mientras que la visualización de la segunda influye en la aplicación de resultados propios de la trigonometría.

- Los alumnos del máster emiten respuestas más directas, alcanzan mayores porcentajes de corrección y, en algunos casos, una mayor variedad de modelos de resolución, sobre todo, para las primeras preguntas. Sólo un alumno presenta construcciones propias con GeoGebra. Los alumnos de este grupo utilizan las tres figuras del test de forma selectiva, seguramente, aquella que para un ítem concreto les parece la más adecuada. Igual que en el grupo 1, tras el análisis de las respuestas emitidas, se puede afirmar que el contenido matemático asociado al pentágono regular forma parte del conocimiento común y del horizonte matemático del profesorado, pero estos alumnos no están especializados en este contenido, porque se observa que no alcanzan la coordinación gráfico-aritmética a través de interpretaciones aritmético-algebraicas rigurosas de la visualización de la construcción.

\section{ReFERENCIAS BIBLIOGRÁFICAS}

Ball, D. L., Thames, M. H., y Phelps, G. (2008). Content knowledge for teaching: What makes its special? Journal of Teacher Education, 59(5), 389-407.

Bataille, M. (2009). Another compass-only construction of the golden section and of the regular pentagon. Forum Geom., 8, 167-169.

De Guzmán, M. (1996). El rincón de la pizarra. Madrid: Pirámide.

Duval, R. (1995). Figures Geométriques et discours mathématique. En Sémiosis et pensée humaine: registres sémiotiques et apprentissages intellectuels (pp. 173207). New York: Peter Lang.

Eisenmann, P., Kopka, J., Ondrušová, J., y Přibyl, J. (2013). The strategy of reformulation of a problem. En M. Billich (Ed.), Mathematica IV. Proceedings of the Polish-Czech-Slovak mathematical conference, Catholic University (pp. 31-36). Ružomberok: Verbum, Catholic University in Ružomberok Press.

Fernández, T. (2013). La investigación en visualización y razonamiento espacial. Pasado, presente y futuro. En A. Berciano, G. Gutiérrez, N. Climent y A. Estepa (Eds.), Investigación en Educación Matemática XVII (pp. 19-42). Bilbao: Sociedad Española de Investigación en Educación Matemática (SEIEM). 
Hofsteter, K. (2013). A simple compass-only construction of the regular pentagon. Forum Geom., 8, 147-148.

Jakobsen, A., Thames, M. H., y Ribeiro, M. (2013). Delineating issues related to Horizon Content Knowledge for mathematics teaching. En B. Ubuz y M.A. Mariotti (Eds.), Actas del CERME 8 (pp. 3055-3064). Antalya, Turquía: ERME.

Lord, N. (2010). From hexagon to pentagon. SYMmetryplus, 38, 14.

Miles, D., y Pritchard, C. (2009). Three trigonometric results from a regular pentagon. Math. Sch. (Leicester), 38(1), 33-34.

Presmeg, N. C. (1997). Generalization using imagery in mathematics. En L. D. English (Ed.), Mathematical Reasoning: analogies, metaphors and metonymies in mathematics learning (pp. 299-312). Mahwah, NJ: Erlbaum.

Presmeg, N. C. (2006). Research on visualization in learning and teaching mathematics. En A. Gutiérrez y P. Boero (Eds.), Handbook of research on the psychology of mathematics education: Past, present and future (pp. 205-235). Rotterdam, The Netherlands: Sense Publishers.

Presmeg, N. (2011). Overcoming Pedagogical Barriers Associated with Exploratory Tasks in a College Geometry Course. En O. Zaslavsky y P. Sullivan (Eds.), Constructing Knowledge for Teaching Secondary Mathematics (pp. 279-290). New York: Springer.

Pritchard, C. (2013). Fibonacci pegs and an angel theorem. Math. Sch. (Leicester), 41(5), 10-11.

Schoenfeld, A. (2010). How we think. New York: Routledge.

Shulman, L. S. (1986). Those who understand. Knowledge growth in teaching. Educational Researcher, 15(2), 4-14. 